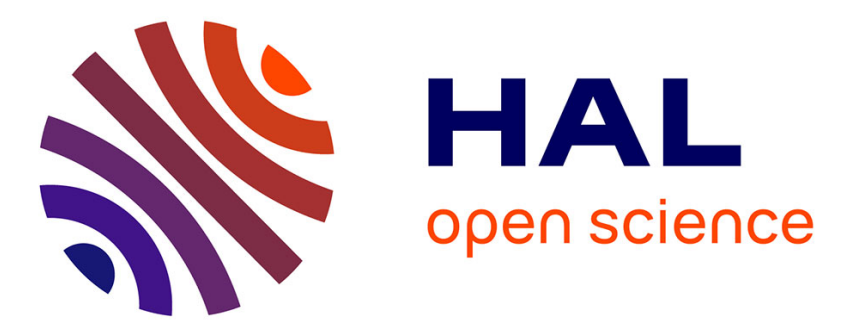

\title{
Pressure dependence of the organic superconductivity in (TMTSF)2PF 6
}

\author{
H.J. Schulz, D. Jérome, M. Ribault, A. Mazaud, K. Bechgaard
}

\section{To cite this version:}

H.J. Schulz, D. Jérome, M. Ribault, A. Mazaud, K. Bechgaard. Pressure dependence of the organic superconductivity in (TMTSF)2PF 6. Journal de Physique Lettres, 1981, 42 (2), pp.51-54. 10.1051/jphyslet:0198100420205100 . jpa-00231871

\section{HAL Id: jpa-00231871 https://hal.science/jpa-00231871}

Submitted on 1 Jan 1981

HAL is a multi-disciplinary open access archive for the deposit and dissemination of scientific research documents, whether they are published or not. The documents may come from teaching and research institutions in France or abroad, or from public or private research centers.
L'archive ouverte pluridisciplinaire HAL, est destinée au dépôt et à la diffusion de documents scientifiques de niveau recherche, publiés ou non, émanant des établissements d'enseignement et de recherche français ou étrangers, des laboratoires publics ou privés. 


\title{
Pressure dependence of the organic superconductivity in $(\mathrm{TMTSF})_{2} \mathbf{P F}_{6}$
}

\author{
H. J. Schulz (*), D. Jérome, M. Ribault, A. Mazaud \\ Laboratoire de Physique des Solides (**), Bât. 510, Université Paris-Sud, 91405 Orsay, France \\ and K. Bechgaard \\ H. C. Oersted Institute, Universitetsparken 5, DK 2100 Copenhagen, Denmark
}

(Reçu le 27 octobre 1980, accepté le 27 novembre 1980)

\begin{abstract}
Résumé. - Les mesures de résistivité effectuées sur le composé (TMTSF) ${ }_{2} \mathrm{PF}_{6}$ font apparaître une décroissance très appréciable de la transition supraconductrice sous pression. Ce phénomène est difficile à interpréter avec les théories habituellement utilisées dans les supraconducteurs à bandes étroites. Nous proposons de relier la dépendance en pression de la supraconductivité au mode mou qui est à l'origine de la transition métal-isolant existant à basse pression.
\end{abstract}

\begin{abstract}
High pressure resistivity measurements reveal a very large decrease of the superconducting transition temperature of (TMTSF) ${ }_{2} \mathrm{PF}_{6}$ with pressure. It is difficult to explain this phenomenon with current theories of narrow band superconductors. An explanation in terms of the soft mode of the low pressure metal to insulator transition is proposed.
\end{abstract}

The existence of bulk superconductivity in the quasi-one-dimensional (Q-1-D) organic conductor $(\mathrm{TMTSF})_{2} \mathrm{PF}_{6}$ at high pressure has been unambiguously established by resistive [1] as well as coupled low frequency susceptibility and critical current measurements [2].

According to reference [1] high pressure is required for the observation of superconductivity, and, indeed at pressures lower than about $9 \mathrm{kbar},(\mathrm{TMTSF})_{2} \mathrm{PF}_{6}$ undergoes a metal to insulator transition at low temperature as shown by thermopower and resistivity experiments [3].

Quasi-one-dimensionality of the electron energy spectrum, namely the existence of open Fermi surfaces influences the properties of the superconducting state in several ways. First, below $T_{\mathrm{s}}$, the upper critical field exhibits an anisotropy which reflects the band structure anisotropy [4]. Second, above $T_{\mathrm{s}}$, the high conductivity and the large transverse magnetoresistance existing up to $40 \mathrm{~K}$ can be quantitatively understood in terms of one-dimensional enhanced paraconductivity [5]. However, at present there is no entirely satisfactory microscopic explanation of the

$\left(^{*}\right)$ Present address : I. Institut für Theoretische Physik, Universität Hamburg, 2000 Hamburg 36, W. Germany.

$(* *)$ Laboratoire associé au C.N.R.S. Work supported in part by a DGRST contract $\mathrm{n}^{\circ}$ 80.7.0165. superconducting state occurring under pressure. The purpose of this study is to present new experimental results and suggestions which may promote in the future a better understanding of organic superconductivity. We have investigated superconductivity of (TMTSF) ${ }_{2} \mathrm{PF}_{6}$ at the maximum hydrostatic pressure $(P=24 \mathrm{kbar})$ which we can achieve at present in a beryllium-copper alloy pressure vessel at very low temperatures $(T<0.1 \mathrm{~K})$. The single crystals used in this work are similar to those studied previously [1]. After calibration of the applied load versus hydrostatic pressure, a pressure of $24 \mathrm{kbar}$ is applied at room temperature. The pressure medium (isopentane) freezes under $24 \mathrm{kbar}$ at $300 \mathrm{~K}$. Therefore, the cooling of the pressure vessel is made with a solidified pressure medium from room temperature. This procedure produces very stable contact resistances between the electrical leads and the samples. Two samples were studied simultaneously with two independent electrical circuits. At $24 \mathrm{kbar}$, the conductivity increases between $300 \mathrm{~K}$ and $1.2 \mathrm{~K}$ by a factor of 260 and the low temperature conductivity is about $6 \times 10^{5}(\Omega . \mathrm{cm})^{-1}$ prior to the superconductivity which is observed at $T_{\mathrm{s}}=0.19 \mathrm{~K}$ (Fig. 1).

The application of a small transverse magnetic field induces a shift of the $R$ versus $T$ curve down in temperature and allows the determination of a critical field (Fig. 2). Superconductivity is drastically 


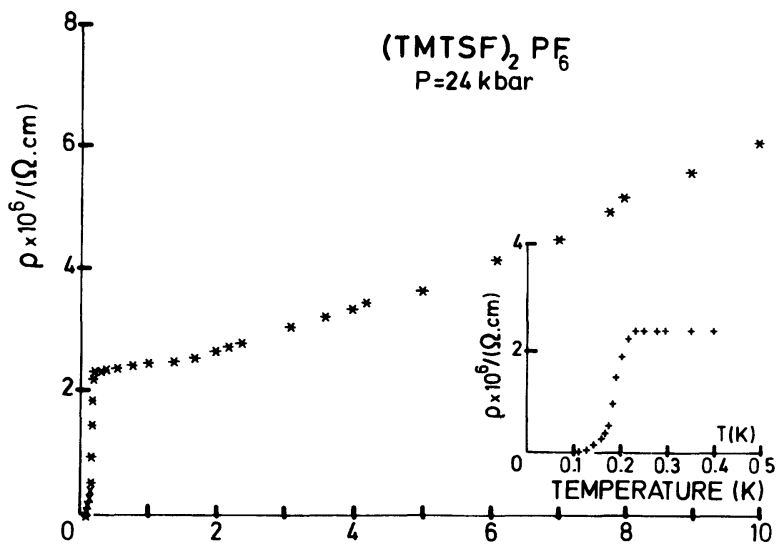

Fig. 1. - Temperature dependence of the resistivity of $(\mathrm{TMTSF})_{2} \mathrm{PF}_{6}$ at $24 \mathrm{kbar}$.

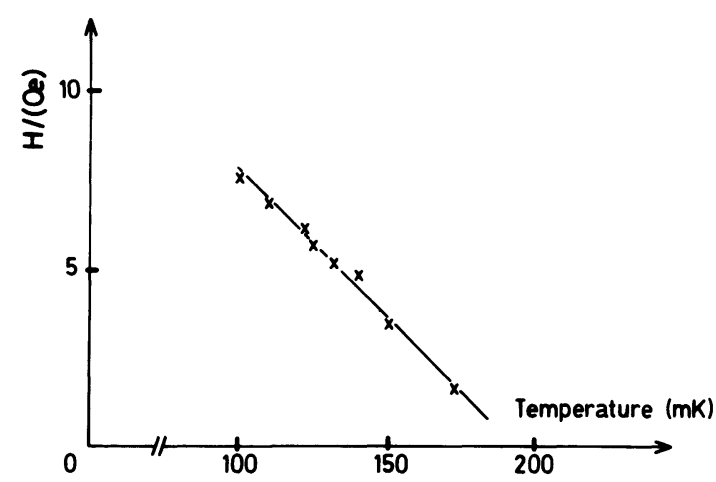

Fig. 2. - Temperature dependence of the transverse critical field of (TMTSF $)_{2} \mathrm{PF}_{6}$ at $24 \mathrm{kbar}$.

suppressed by increase of pressure, suggesting that a reduction in pressure will increase $T_{\mathrm{s}}$. However below 9 kbar superconductivity is inhibited by the occurrence of a very sharp metal to insulator (M-I) transition (see Fig. 3 for the 6.5, 8 and 9 kbar behaviour of the resistance). The data obtained at $9 \mathrm{kbar}$ are interesting in that down to the lowest temperature attainable at this pressure, namely $1.28 \mathrm{~K}$, neither the M-I nor the superconducting transition are observed. At this pressure the temperature dependence of the resistance is essentially metallic-like, although there may be a very weak minimum at $3 \mathrm{~K}$.

In figure 4 we have plotted the dependence of the transition temperatures, $T_{M-I}$ and $T_{\mathrm{s}}$, on the lattice constant along the packing direction of the molecules. Since the axial compressibility of (TMTSF) ${ }_{2} \mathrm{PF}_{6}$ is not yet known, we have used in figure 4 the compressibility of a typical organic conductor, TTFTCNQ [6]. High pressure studies [7] have indicated very similar compressibilities for both TTF-TCNQ and its selenium analogue TSF-TCNQ.

The shape of the phase diagram in the neighbourhood of 9 kbar is not yet known with great accuracy. A better determination will require further high pressure studies with smaller pressure increments.

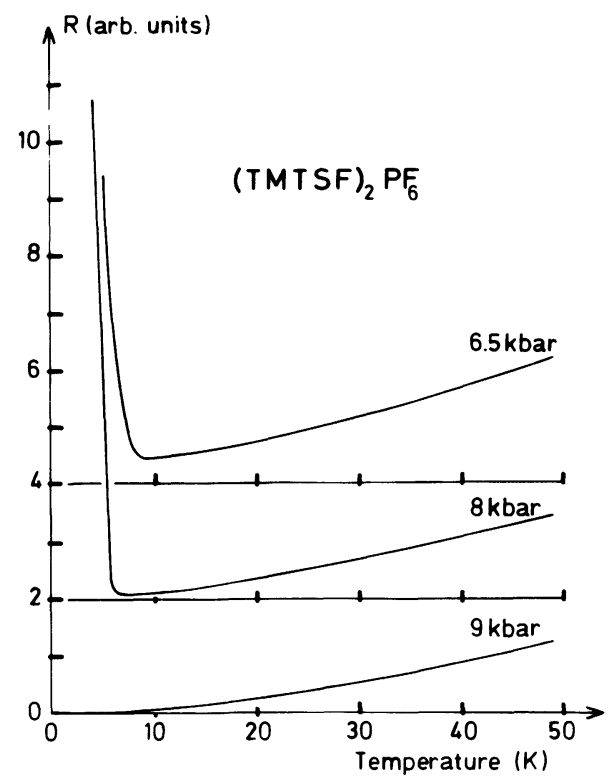

Fig. 3. - Evolution of the resistivity versus temperature curves at several pressures. The results at successive pressures have been shifted by two units.

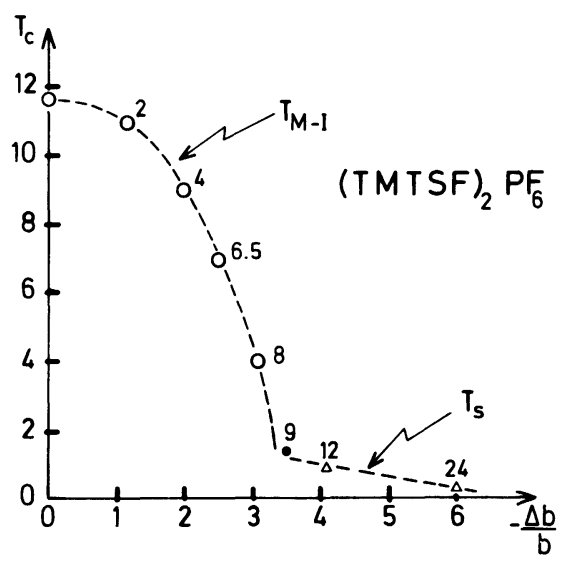

Fig. 4. - Phase diagram of (TMTSF) ${ }_{2} \mathrm{PF}_{6}$ displayed as $T_{\mathrm{c}}$ versus chain-axis lattice constant. The open dots together with the corresponding pressures denote the transition to an insulating state and the open triangles refer to the superconducting transition. The $9 \mathrm{kbar}$ point is not a phase transition point. It simply indicates that no transitions have been detected under these conditions.

However even with these limited data some comments can be made concerning possible transition regions between the insulating and superconducting states. One possibility is a V-shaped phase diagram with no phase transition at all for some critical pressure in the neighbourhood of $9 \mathrm{kbar}$. Such a point of view which follows from some theoretical work $[8,9]$ has been presented in reference [10]. A second possibility is the existence of a first order phase transition line between the insulating and superconducting states [11]. This would imply (for a non vertical transition line) the sequence of phases Metal-Insulator-Superconductor, i.e. reentrant superconductivity. Some effects of coexistence between insulating and superconducting 
instabilities may have been observed in the critical pressure domain [12]. However the insulating instability does not completely develop before the occurrence of superconductivity [13]. This may be explained by non magnetic impurities smearing the M-I transition but leaving the S-transition unaffected.

The behaviour of the upper critical field at $24 \mathrm{kbar}$ in figure 2 can be interpreted in terms of the LandauGinzburg analysis of Josephson coupled chains [4, 5]. Within this model we have :

$$
\left.\frac{\mathrm{d} H_{\mathrm{c}_{2}}^{\perp}}{\mathrm{d} T}\right|_{T_{\mathrm{c}}}=\varphi_{0} / 2 \pi \xi_{\|}(0) \xi_{\perp}(0) T_{\mathrm{s}}
$$

where the zero temperature coherence lengths are related to the band structure anisotropy by :

$$
\xi_{\|} / \xi_{\perp}=t_{\|} / t_{\perp} \text { and } \xi_{\|} \sim v_{\mathrm{F}} / t_{\mathrm{s}}
$$

This implies that $\mathrm{a}_{\mathrm{c}_{2}}^{\perp} /\left.\mathrm{d} T\right|_{T_{\mathrm{s}}}$ becomes proportional to $T_{\mathrm{s}}$. The data of figure 2 , with

$$
\mathrm{d} H_{\mathrm{c}_{2}}^{\perp} /\left.\mathrm{d} T\right|_{T_{\mathrm{s}}}=80 \mathrm{Oe} / \mathrm{K} \text { and } T_{\mathrm{s}}=0.19 \mathrm{~K}
$$

are therefore in fair agreement with the $12 \mathrm{kbar}$ results [1] $\left(\mathrm{d} H_{\mathrm{c}_{2}}^{\perp} /\left.\mathrm{d} T\right|_{T_{\mathrm{s}}}=222 \mathrm{Oe} / \mathrm{K}\right.$ and $\left.T_{\mathrm{s}}=0.9 \mathrm{~K}\right)$.

In the rest of this article we shall try to draw some conclusions concerning the origin of superconductivity in (TMTSF) ${ }_{2} \mathrm{PF}_{6}$, from figure 4.

The decrease of $T_{\mathrm{s}}$ from $0.9 \mathrm{~K}$ at $12 \mathrm{kbar}$ to $0.19 \mathrm{~K}$ at $24 \mathrm{kbar}$ gives a mean pressure dependence of $\partial \ln T_{\mathrm{s}} \partial P \approx-7 \times 10^{-5} \mathrm{bar}^{-1}$. The bandwidth of (TMTSF) ${ }_{2} \mathrm{PF}_{6}$ derived by optical and thermopower results, of about $1 \mathrm{eV}$ [3], suggests its pressure dependence should be compared with that of d-band superconductors.

The pressure dependence of $T_{\mathrm{s}}$ in those substances where the electron-electron attraction is due to acoustic phonons can be ascribed to a combination of three effects [14] : (i) under pressure the acoustic phonon frequency increases, as described by the lattice Gruneisen constant, which leads to a decrease of $T_{\mathrm{s}}$, (ii) the diminished ion-ion distance leads to a larger electron-phonon matrix element, thus increasing $T_{\mathrm{s}}$, (iii) the bandwidth is increased by pressure so that the density of states decreases, but the effect of Coulomb repulsion is also decreased. This bandwidth effect is usually less important than points (i) and (ii).

The combination of these effects may lead either to an increase or a decrease of $T_{\mathrm{s}}$ with pressure, as observed experimentally [15]. Although the Gruneisen constant of (TMTSF) ${ }_{2} \mathrm{PF}_{6}$ has not been measured, for a typical organic conductor, TTF-TCNQ, a value of 3 at $300 \mathrm{~K}$ can be deduced from the pressure dependence of the axial compressibility $[6,16]$, and even lower values (by about a factor two) have been derived at low temperatures [17]. Thus on the one hand we expect the changes of lattice properties of (TMTSF) ${ }_{2} \mathrm{PF}_{6}$ under pressure to be comparable to those of the d-band superconductors. But on the other hand the decrease of $T_{\mathrm{s}}$ with pressure in (TMTSF) ${ }_{2} \mathrm{PF}_{6}$ is larger than the values of decrease of $T_{\mathrm{s}}$ found in d-band superconductors [15] by at least a factor of 10 . Consequently, it seems unlikely that one can explain this large decrease by the same mechanism for the d-band superconductors.

In molecular metals, intramolecular phonons are known to be strongly coupled to the electrons [18] and such modes have been shown to be favourable to superconductivity [19]. However, both the frequency and strength of coupling of these modes to the electrons are molecular properties and are therefore independent of pressure. Thus only point (iii) remains as a possible explanation of the pressure dependence of $T_{\mathrm{s}}$. However, from optical data on TTF-TCNQ [20] and the occurrence of longitudinal commensurability under pressure in TTF-TCNQ [21] and TSFTCNQ [7] the changes of the bandwidth under pressure are shown to be quite small and can surely not account for the variation of $T_{\mathrm{s}}$.

Another possibility for a pressure dependent $T_{\mathrm{s}}$ are changes in the "one-dimensionality" under pressure. In an isolated metallic chain a superconducting phase transition at finite temperature is not possible and in a system of coupled chains a finite interchain tunnelling integral $t_{\perp}$ is necessary to obtain a non zero transition temperature. If only the superconducting transition is considered, current theories $[22,24]$ predict an increase of $T_{s}$ with increasing $t_{\perp}$. As $t_{\perp}$ is increased with pressure one would expect $\partial T_{\mathrm{s}} / \partial P>0$ from this mechanism, in contrast to the experimental result. One should also note that the conductivity anisotropy at room temperature is nearly constant, at least up to $12 \mathrm{kbar}$ [4]. We must therefore rule out changes in the " one-dimensionality " as an origin of the pressure dependence of $T_{\mathrm{s}}$.

In the above discussion we have not considered the presence of a metal-insulator transition with a transition temperature vanishing at a critical pressure of about $9 \mathrm{kbar}$. Although at higher pressures there is a superconducting phase transition instead of the metal-insulator transition, one may reasonably assume that a soft mode corresponding to the metal-insulator transition is still present slightly above the critical pressure and vanishes with increasing pressure. For the case of competition between a charge-density wave (CDW) and superconducting transitions in 1-D conductors, it has been shown that the presence of a soft mode enhances $T_{\mathrm{s}}[8,9,25]$, so that vanishing of the soft mode may lead to a rapid decrease of $T_{\mathrm{s}}$, as observed experimentally. This explanation does not of course exclude acoustic or intramolecular phonons from playing a role in the superconducting transition.

Moreover, it has been suggested that the low pressure insulating phase [26] is a spin-density wave state (SDW) which indicates spin-density fluctuations instead of a coupled charge density-lattice modulation [27] (soft phonons) as the soft mode, i.e. an 
electron-electron interaction not mediated by the lattice. Furthermore, the presence of a SDW means that one is in the $g_{1}>0$ part of the usual $g_{1}, g_{2}$ phase diagram [28] and the transition to the superconducting also takes place in that part of the diagram, as suggested by Barišić and Brazovskii [29]. Although the triplet superconducting response function is more strongly divergent than the singlet function [30] for $g_{1}>0$, a triplet superconducting phase is extremely sensitive to impurities, so that impurities tend to stabilize the singlet phase. Experimentally it is found that $T_{\mathrm{s}}$ is independent of the sample purity (where $T_{\mathrm{s}}$ is derived from the width of the superconducting transition) so that it seems unlikely that the superconducting phase is of the triplet type. In conclusion, the most likely reasonable explanation for the strong pressure dependence of $T_{\mathrm{s}}$ is by the presence of a soft mode, possibly a SDW fluctuation, which would enhance $T_{\mathrm{s}}$. The vanishing of this soft mode with increasing pressure would then result in a drop in $T_{\mathrm{s}}$

Acknowledgments. - We would like to thank G. Benedek for his help in pressure experiments. We have had very fruitful discussions with J. Friedel and S. Barišić. One of us (H.J.S.) thanks J. Appel for helpful conversations.

\section{References}

[1] JÉrome, D., Mazaud, A., Ribault, M. and BechgaARd, K., J. Physique Lett. 41 (1980) L-95.

[2] Ribault, M., Benedek, G., Jérome, D. and BechgaArd, K., J. Physique Lett. 41 (1980) L-397.

[3] Bechgaard, K., Jacobsen, C. S., Mortensen, K., PederSEN, H. J. and ThORUP, N., Solid State Commun. 33 (1980) 1119.

[4] Jérome, D., to be published in Chemica Scripta, Proceedings of the International Conference on "Low Dimensional Synthetic Metals " Helsingör, Denmark, August 1980, and Jérome, D. and Schulz, H. J., to be published in Physics in One Dimension, editor J. Bernasconi (Springer) 1980.

[5] Schulz, H. J., Jérome, D., Mazaud, A., Ribault, M. and BECHGAARD, K., preprint (1980).

[6] Debray, D., Millet, R., Jérome, D., Barišić, S., Giral, L. and FABre, J. M., J. Physique Lett. 38 (1977) L-227.

[7] Thomas, J. F. and JÉrome, D., Solid State Commun. (1980), in press.

[8] Prigodin, V. N. and Firsov, Yu. A., JETP Lett. 25 (1977) 79 and Sov. Phys. JETP 49 (1979) 813.

[9] Suzumura, Y. and Fukayama, H., J. Low. Temp. Phys. 31 (1978) 273.

[10] JÉROME, D., presented at the International Conference on « Low Dimensional Synthetic Metals " Helsingör, Denmark, August 1980, to be published in Chemica Scripta.

[11] Private Communication from J. Friedel.

[12] GReene, R. L. and ENGLeR, E. M., preprint.

Andres, K., Wudl, F., MCWhan, D. B., Thomas, G., NaleWAJEK, D. and STEVEnS, A. L., preprint.

[13] Preliminary results obtained with the system (TMTSF) ${ }_{2} \mathrm{AsF}_{6}$ indicate such a behaviour.
[14] Garland, J. W. and Bennemann, K. H., in Superconductivity in $d$ - and $f$-Band Metals, ed. by D. H. Douglass (American Institute of Physics, New York) 1972, p. 255.

[15] SMITH, F. F., in reference [14], p. 293.

[16] Debray, D., Jérome, D. and Barišić, S., High Pressure and Low Temperature Physics, edited by C. W. Chu and J. A. Woollam (Plenum Press) 1978, p. 565.

[17] We thank S. MegTeRT for a discussion of his recent data on the low temperature compressibility of TTF-TCNQ.

[18] Rice, M. J. and Lipari, N. O., Phys. Rev. Lett. 38 (1977) 437.

[19] Horovitz, B., Phys. Rev. B 16 (1977) 3843.

[20] Welber, B., Seiden, P. E. and Grant, P. M., Phys. Rev. B 18 (1978) 2692.

[21] Friend, R. H., Miljak, M. and Jérome, D., Phys. Rev. Lett. 40 (1978) 1048.

[22] Klemm, R. S. and Gutfreund, H., Phys. Rev. B 14 (1976) 1086.

[23] Larkin, A. I. and SaK, J., Phys. Rev. B 18 (1978) 6053.

[24] BulaeVSKI, L. N. and BuzdiN, A. I., preprint (1980).

[25] Gutrreund, H., Horovitz, B. and Weger, M., J. Phys. C 7 (1974) 383.

[26] Walsh, W. M., Wudl, F., Thomas, G. A., NalewajeK, D., Hauser, J. J., Lee, P. A. and Poehler, T., Phys. Rev. Lett. 45 (1980) 829.

[27] Pouget, J. P., in Proceedings of the International Conference on "Low Dimensional Synthetic Metals » Helsingör, August 1980, to be published in Chemica Scripta.

[28] For a review see Solyom, J., Adv. Phys. 28 (1979) 201.

[29] Barišíc, S. and BrazovskiI, S. A., Proc. EPS Conference, Antwerp 1980 and BARIŠÍ, S., preprint (1980).

[30] Fukuyama, H., Rice, T. M., Varma, C. M. and Halperin, B. I., Phys. Rev. B 10 (1974) 3775. 